\title{
A guide to prospective meta-analysis
}

\section{Check for updates}

${ }^{1}$ NHMRC Clinical Trials Centre, University of Sydney, Locked bag 77, Camperdown NSW 1450, Australia

${ }^{2}$ National Health and Medical Research Council, Canberra, Australia

${ }^{3}$ Johnson \& Johnson, Titusville, NJ, USA

Correspondence to: A L Seidler lene.seidler@ctc.usyd.edu.au (ORCID 0000-0002-0027-1623) Additional material is published online only. To view please visit the journal online.

Cite this as: $B M$ J 2019;367:15342 http://dx.doi.org/10.1136/bmj.15342

Accepted: 8 August 2019

\author{
Anna Lene Seidler, ${ }^{1}$ Kylie E Hunter, ${ }^{1}$ Saskia Cheyne, ${ }^{1}$ Davina Ghersi, ${ }^{1,2}$ Jesse A Berlin, ${ }^{3}$ \\ Lisa Askie $^{1}$
}

In a prospective meta-analysis (PMA), study selection criteria, hypotheses, and analyses are specified before the results of the studies related to the PMA research question are known, reducing many of the problems associated with a traditional (retrospective) meta-analysis. PMAs have many advantages: they can help reduce research waste and bias, and they are adaptive, efficient, and collaborative. Despite an increase in the number of health research articles labelled as PMAs, the methodology remains rare, novel, and often misunderstood. This paper provides detailed guidance on how to address

\section{SUMMARY POINTS}

In a prospective meta-analysis (PMA), studies are identified and determined to be eligible for inclusion before the results of the studies related to the PMA research question are known

PMAs are applicable to high priority research questions where limited previous evidence exists and where new studies are expected to emerge

Compared with standard systematic review and meta-analysis protocols, key adaptations should be made to a PMA protocol, including search methods to identify planned and ongoing studies, details of studies that have already been identified for inclusion, core outcomes to be measured by all studies, collaboration management, and publication policy

A systematic search for planned and ongoing studies should precede a PMA, including a search of clinical trial registries and medical literature databases, and contacting relevant stakeholders in the specialty

PMAs are ideally conducted by a collaboration or consortium, including a central steering and data analysis committee, and representatives from each individual study

Usually PMAs collect individual participant data, but PMAs of aggregate data are also possible. PMAs can include interventional or observational studies

PMAs can enable harmonised collection of core outcomes, which can be particularly useful for rare but important outcomes, such as adverse side effects Adaptive forms of PRISMA (preferred reporting items for systematic reviews and meta-analyses) and quality assessment approaches such as GRADE (grading of recommendations assessment, development, and evaluation) should be used to report and assess the quality of evidence for a PMA. The development of a standardised set of reporting guidelines and PMA specific evidence rating tools is highly desirable

PMAs can help to reduce research waste and bias, and they are adaptive, efficient, and collaborative the key elements for conducting a high quality PMA with a case study to illustrate each step.

Systematic reviews and meta-analyses of the best available evidence are widely used to inform healthcare policy and practice. ${ }^{12}$ Yet the retrospective nature of traditional systematic reviews and meta-analyses can be problematic. Positive results are more likely to be reported and published (phenomena known as selective outcome reporting and publication bias), and therefore including only published results in a metaanalysis can produce misleading results ${ }^{3}$ and pose a threat to the validity of evidence based medicine. ${ }^{4}$ In the planning stage of a traditional meta-analysis, knowledge of individual study results can influence the study selection process as choosing the key components of the review question and eligibility criteria might be based on one or more positive studies. ${ }^{25}$ Meta-analyses on the same topic can reach conflicting conclusions because of different eligibility criteria. $^{2}$ Also, inconsistencies across individual studies in outcome measurement and analyses can make the combination of data difficult. ${ }^{6}$

Prospective meta-analyses (PMAs, see box 1) have recently been described as next generation systematic reviews $^{7}$ that reduce the problems of traditional retrospective meta-analyses. Ioannidis and others even argue that "all primary original research may be designed, executed, and interpreted as prospective meta-analyses." 9 For PMAs, studies are included prospectively, meaning before any individual study results related to the PMA research question are known. ${ }^{10}$ This reduces the risk of publication bias and selective reporting bias and can enable better harmonisation of study outcomes.

The number of meta-analyses described as PMAs is increasing (fig 1). But the definition, methodology, and reporting of previous PMAs vary greatly, and guidance on how to conduct them is limited, outdated, and inconsistent. ${ }^{11}{ }^{12}$ With recent advancements in computing capabilities, and the ability to identify planned and ongoing studies through increased trial registration, the planning and conduct of PMAs have become more efficient and effective. For PMAs to be successfully implemented in future health research, a revised PMA definition and expanded guidance are required. In this article, we, the Cochrane PMA Methods Group, present a step by step guide on how to perform a PMA. Our aim is to provide up to date guidance on the key principles, rationale, methods, and challenges for each step, to enable more researchers to understand and use this methodology successfully. Figure 2 shows a summary of the steps needed to perform a PMA. 


\section{Box 1: Definition of a prospective meta-analysis}

The key feature of a prospective meta-analysis (PMA) is that the studies or cohorts are identified as eligible for inclusion in the meta-analysis, and hypotheses and analysis strategies are specified, before the results of the studies or cohorts related to the PMA research question are known

\section{Case study: Neonatal Oxygenation Prospective Meta-analysis (NeOProM)}

We will illustrate each step with an example of a PMA of randomised controlled trials conducted by the Neonatal Oxygenation Prospective Meta-analysis (NeOProM) Collaboration. ${ }^{13}$ In this PMA, five groups prospectively planned to conduct separate, but similar, trials assessing different target ranges for oxygen saturation in preterm infants, and combine their results on completion. Although no difference was found in the composite primary outcome of death or major disability, a statistically significant reduction in the secondary outcome of death alone was found for the higher oxygen target range, but no change in major disability. This PMA resolved a major debate in neonatology.

Steps for performing a prospective meta-analysis Step 0: deciding if a PMA is the right methodology PMA methodology should be considered for a high priority research question for which new studies are expected to emerge and limited previous evidence exists (fig 3):

Priority research question-PMAs should be planned for research questions that are a high priority for healthcare decision makers. Ideally, these questions should be identified using priority setting methods within consumer-clinician collaborations, and/or they should address priorities identified by guideline committees, funding bodies, or clinical and research associations. Often these questions are in areas where important new treatment or prevention strategies have recently emerged, or where practice varies because of insufficient evidence.

New studies expected-PMAs are only feasible if new studies are likely to be included-for example, if the research question is an explicit priority for funding

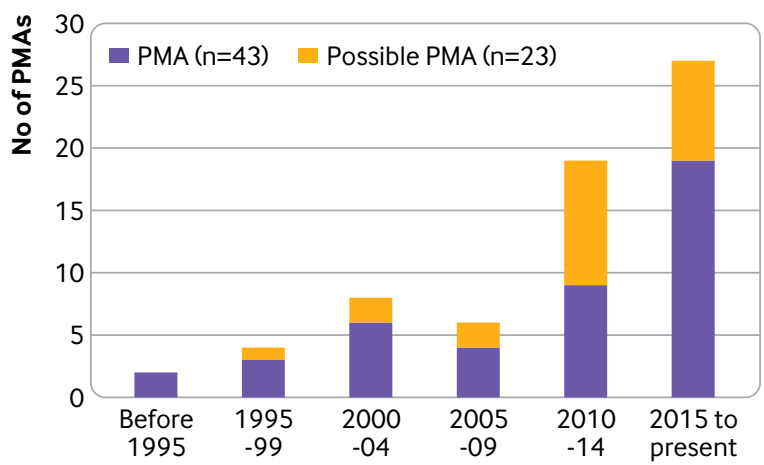

Fig 1 | Number of prospective meta-analyses (PMAs) over time. Possible PMA describes studies that seem to fulfil the criteria for a PMA but not enough information was reported to make a definite decision on their status as a PMA. These data are based on a systematic search of the literature (see appendix 1 for methodology) bodies or research associations. Some PMAs have been initiated after researchers learnt they were planning or conducting similar studies, and so they decided to collaborate and prospectively plan to combine their data. In other cases, a research question is posed by a consortium of investigators who then decide to plan similar studies that are combined on completion. A research team planning a PMA can play an active role in motivating other researchers to conduct similar studies addressing the same research question. A PMA can therefore be a catalyst for initiating a programme of priority research to answer important questions. ${ }^{8}$ Initiating a PMA rather than conducting a large multicentre study can be advantageous as PMAs allow flexibility for each study to answer additional local questions, and the studies can be funded independently, which circumvents the problem of funding a mega study.

Insufficient previous evidence-A PMA should only be conducted if insufficient evidence exists to answer the research question. If sufficient evidence is available (eg, based on a retrospective meta-analysis), no further studies and no PMA should be planned, to avoid research waste.

If evidence is available, but is insufficient for clinical decision making, a nested PMA should be considered. A nested PMA integrates prospective evidence into a retrospective meta-analysis, making best use of existing and emerging evidence while also retaining some benefits of PMAs. A nested PMA allows the assessment of publication bias and selective reporting bias by comparing prospectively included evidence with retrospective evidence in a sensitivity analysis. Studies that are prospectively included can be harmonised with other ongoing studies, and with previous related retrospective studies, to optimise evidence synthesis (see step 5).

\section{Case study}

PMA methodology was chosen to determine the optimal target range for oxygen saturation in preterm infants for several reasons:

Priority research question-oxygen has been used to treat preterm infants for more than 60 years. The different oxygen saturation target ranges used in practice have been associated with clinically important outcomes, such as mortality, disability, and blindness. Changing the oxygen saturation target range would be relatively easy to implement in clinical practice.

Insufficient previous evidence-evidence was mainly observational, with no recent, high quality randomised controlled trials available.

New studies expected-a total sample size of about 5000 infants was needed to detect an absolute difference in death or major disability of $4 \%$. The NeOProM PMA was originally proposed as one large multicentre, multinational trial. ${ }^{14}$ But because expensive masked pulse oximeters were needed, one funder could not support a study of sufficient sample size to reliably answer the clinical question. Instead, a PMA collaboration was initiated. Each group of NeOProM 


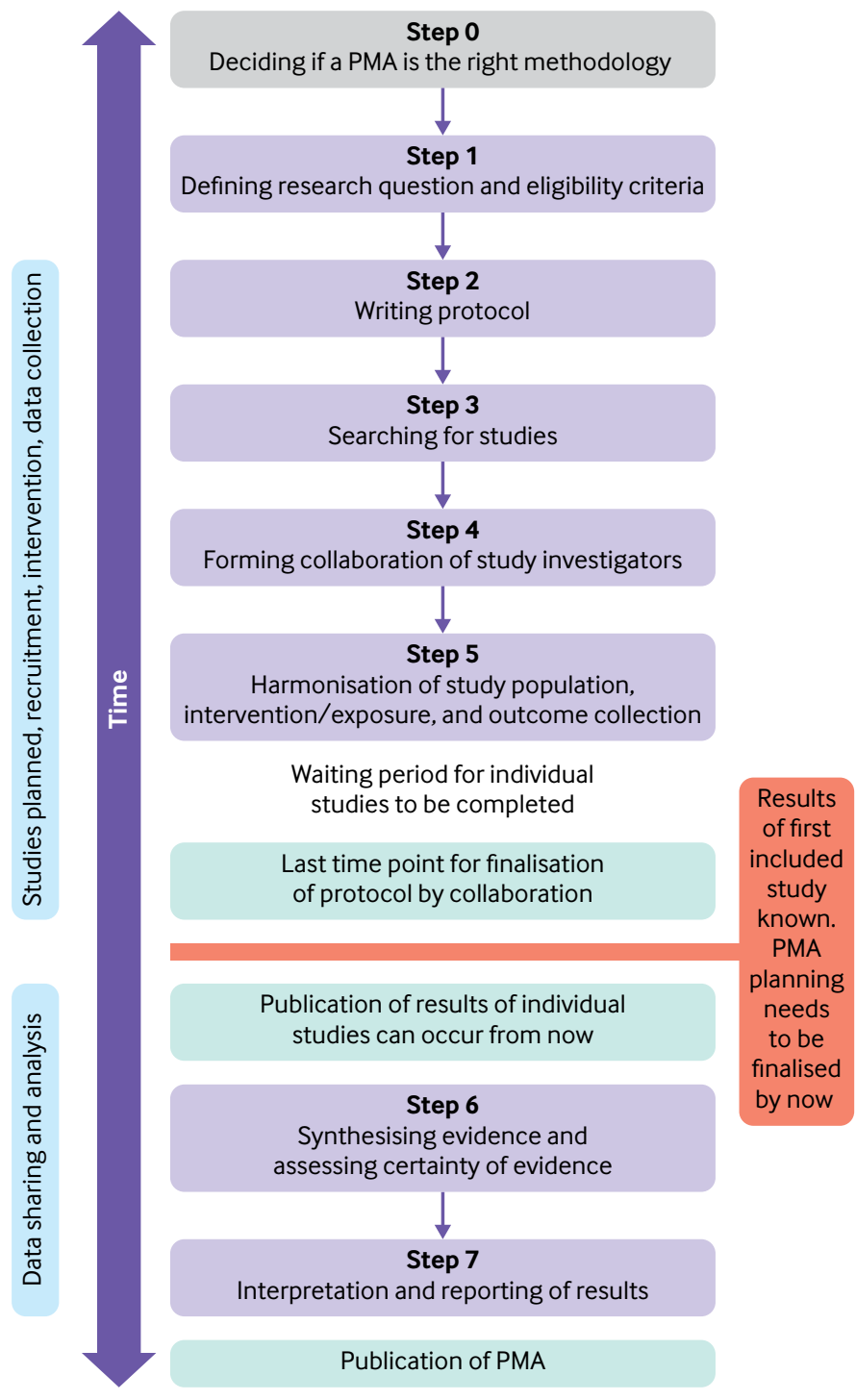

Fig 2 | Steps in conducting a prospective meta-analysis (PMA)

investigators obtained funding to conduct their own trial (although alone each study was underpowered to answer the main clinical question), could choose their own focus, and publish their own results, but with agreement to contribute data to the PMA to ensure sufficient combined statistical power to reliably detect differences in important outcomes.

\section{Step 1: defining the research question and the eligibility criteria}

At the start of a PMA, a research question needs to be specified. Research questions for PMAs should be formed in a similar way to traditional retrospective systematic reviews. Guidance for formulating a review question is available in the Cochrane Handbook for Systematic Reviews of Interventions. ${ }^{15}$ For PMAs of interventional studies, the PICO system (population, intervention, comparison, outcome) should be used. To avoid selective reporting bias, the PMA research question and hypotheses need to be specified before any study results related to the PMA research questions are known.

PMAs are possible for a wide range of different study types-their applicability reaches beyond randomised controlled trials. An interventional PMA includes interventional studies (eg, randomised controlled trials or non-randomised studies of interventions). For interventional PMAs, the key inclusion criterion of "no results being known" usually means that the analyses have not been conducted in any of the trials included in the PMA.

An observational PMA includes observational studies. For observational PMAs, "no results being known" would mean that no analyses related to the PMA research question have been done. As many observational studies collect data on different outcomes, a meta-analysis can be classified as a PMA if unrelated research questions have already been analysed before inclusion in the PMA. For instance, for a PMA on the risk of lung cancer for people exposed to air pollution, observational studies where the relation between cardiovascular disease and air pollution has already been analysed can be included in the PMA, but only if the analyses on the association between lung cancer and air pollution have not been done. In this case, however, little harmonisation of outcome collection is possible (unless the investigators agree to collect additional data).

\section{Case study}

The NeOProM PMA addressed the research question, does targeting a lower oxygen saturation range in extremely preterm infants, from birth or soon after, increase or decrease the composite outcome of death or major disability in survivors by $4 \%$ or more?

The PICOS system was applied to define the eligibility criteria:

- Participants=infants born before 28 weeks' gestation and enrolled within 24 hours of birth

- Intervention=target a lower (85-89\%) oxygen saturation $\left(\mathrm{SpO}_{2}\right)$ range

- $\quad$ Comparator=target a higher (91-95\%) $\mathrm{SpO}_{2}$ range

- $\quad$ Outcome=composite of death or major disability at a corrected age of 18-24 months

- Study type=double blinded, randomised controlled trial (making this an interventional PMA).

\section{Step 2: writing the protocol}

Key elements of the protocol need to be finalised for the PMA before any individual study results related to the PMA research question are known. These include specification of the research questions, eligibility criteria for inclusion of studies, hypotheses, outcomes, and the statistical analysis strategy. The preferred reporting items for systematic reviews and meta-analyses extension for protocols (PRISMA-P) ${ }^{16}$ provides some guidance on what should be included. As these reporting items were created for retrospective meta-analyses, however, key adaptations need to be made for PMA protocols (see box 2). 


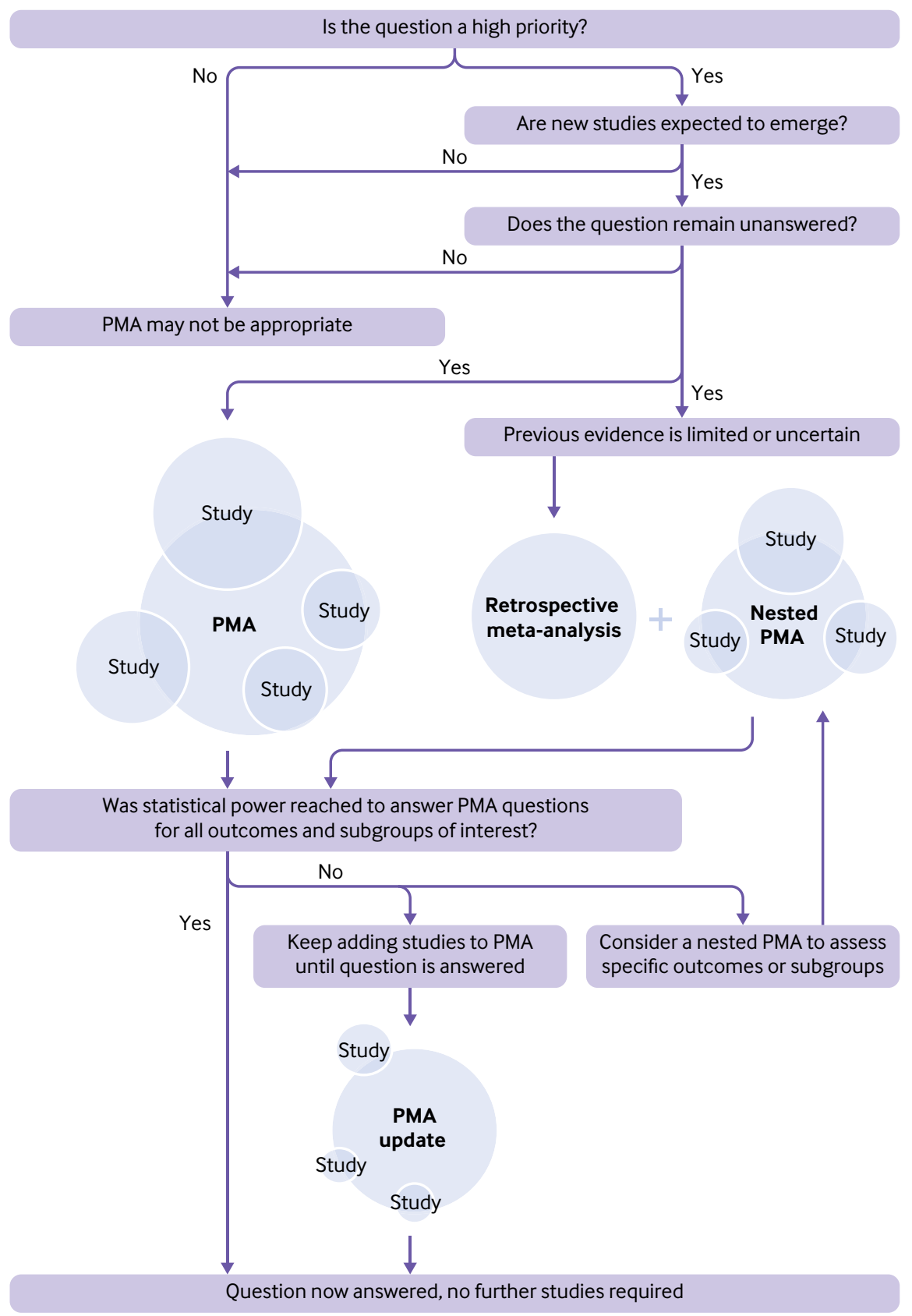

Fig 3 | When to conduct a prospective meta-analysis (PMA)

An initial PMA protocol should be drafted before the search for eligible studies, but it can be amended after searching and after all studies have been included if the results of the included studies are not known when the PMA protocol is finalised. The investigators of the included studies can agree on the collection and analysis of additional rare outcomes and these outcomes can be included in a revised version of the protocol.

The final PMA protocol should be publicly available on the international prospective register of systematic reviews, PROSPERO ${ }^{17}$ (which supports registration of PMAs), before the results (relating to the PMA research question) of any of the included studies are known. A full version of the PMA protocol can be published in a peer reviewed journal or elsewhere.

\section{Case study}

For the NeOProM PMA, an initial protocol was drafted by the lead investigators and discussed and refined by collaborators from all the included trials. The PMA protocol was registered on ClinicalTrials.gov in 2010 (NCT01124331) because PROSPERO had not yet been launched. After the launch of PROSPERO in 2011, the protocol was registered (CRD42015019508). The full version of the protocol was published in $B M C$ Pediatrics. $^{18}$ 


\section{Box 2: Key additional reporting items for a PMA protocol}

For a PMA, several key items should be reported in the protocol in addition to PRISMA-P items:

\section{Search methods}

The search methods need to include how planned and ongoing studies are identified and how potential collaborators will be or have been contacted to participate (see step 3)

\section{Study details}

Details for studies already identified for inclusion should be listed, along with a statement that their results related to the PMA research question are not yet known (see step 1)

\section{Core outcomes}

Any core outcomes that will be measured by all the included studies should be specified, along with details on how and why they should be measured, to facilitate outcome harmonisation (see step 5)

\section{Type of data collected}

PMAs often collect individual participant data (that is, row by row data for each participant) but they may also collect aggregate data (that is, summary data for each study), and some combine both (see step 6)

\section{Collaboration management and publication policy}

Collaboration management and publication policy (see steps 4 and 7) should be specified, including details of any central steering and data analysis committees

\section{Step 3: searching for studies}

After the PMA protocol is finalised, a systematic literature search is conducted, similar to that of a systematic review for a high quality meta-analysis. The main resources available for identifying planned and ongoing studies are clinical trial registries. Currently, 17 global clinical trial registries provide data to the World Health Organization's International Clinical Trials Registry Platform. ${ }^{19}$ Views on the best strategies for searching trial registries differ. ${ }^{20}$ Limiting the search by date can be useful (eg, only studies registered within a reasonable time frame, taking into account the expected study duration and follow-up times) to reduce the search burden and exclude studies registered earlier that would likely be completed and thus ineligible for a PMA. Ideally, searches should be repeated on a regular basis to identify new eligible studies.

Prospective trial registration is mandated by various legislative, ethical, and regulatory bodies but compliance is not complete. ${ }^{21-23}$ Observational studies are not required to be registered. Hence additional approaches to identifying planned and ongoing studies should be pursued, including searching bibliographic databases for conference abstracts, study protocols, and cohort descriptions, and approaching relevant stakeholders. The existence and possibility of joining the PMA can be publicised through the publication of PMA protocols, presentations at relevant conferences and research forums, and through an online presence (eg, a collaboration website).

\section{Case study}

For NeOProM, the Cochrane Central Register of Controlled Trials, Medline through PubMed, Embase, and CINAHL, clinical trial registries (using the WHO portal (www.who.int/ictrp/en/) and ClinicalTrials. gov), conference proceedings, and the reference lists of retrieved articles were searched. Key researchers in the specialty were contacted to inquire if they were aware of additional trials. The abstracts of the relevant perinatal meetings (including the Neonatal Register and the Society for Paediatric Research) were searched using the keywords "oxygen saturation". Five planned or ongoing trials meeting the inclusion criteria for the NeOProM PMA were identified, based in Australia, New Zealand, Canada, the United Kingdom, and the United States. The trials completed enrolment and follow-up between 2005 and 2014 and recruited a total of 4965 preterm infants born before 28 weeks' gestation. No results for any of the trials were known at the time each trial agreed to be included in the PMA. All the NeOProM trials were identified by discussion with collaborators, and no additional trials were identified from electronic database searches.

\section{Step 4: forming a collaboration of study investigators}

Ideally, PMAs are conducted by a collaboration or consortium, including a central steering committee (leading the PMA and managing the collaboration), a data analysis committee (responsible for data management, processing, and analysis), and representatives from each study (involved in decisions on the protocol, analysis, and interpretation of the results). Regular collaboration meetings can be beneficial for achieving consensus on disagreements and in keeping study investigators involved in the PMA process. Transparent processes and a priori agreements are crucial for building and maintaining trust within a PMA collaboration.

Investigators might refuse to collaborate. Refusal to collaborate is less likely in a PMA than in a retrospective individual participant data meta-analysis as reaching agreement to share data is easier if studies are in their planning phases and can still be amended and harmonised after internal discussions. Aggregate data can be included in the PMA even if investigators refuse to collaborate, if the relevant summary data can be extracted from the resulting publications when the studies are completed. The ability to harmonise studies (step 5), however, may be limited if eligible investigators refuse to participate.

\section{Case study}

The NeOProM Collaboration comprised at least one investigator and a statistician from each of the included trials, and a steering group. All investigators and the steering group agreed on key aspects of the protocol before the results of the trials were known, and they also developed and agreed on a common data collection form, coding sheet, and detailed analysis plan. The NeOProM Collaboration met regularly by teleconference, and at least once a year face to face, to reach consensus on disagreements and to discuss the progress of individual trials, funding, data harmonisation, analysis plans, and interpretation of the PMA findings. 
Step 5: harmonisation of included study population, intervention/exposure, and outcome collection

When a collaboration of investigators of planned or ongoing studies has been formed, the investigators can work together to harmonise the design, conduct, and outcome collection of the included studies to facilitate a meta-analysis and interpretation. A common problem with retrospective meta-analyses is that interventions are administered slightly differently across studies, or to different populations, and outcome collection, measurement, or reporting can differ. These differences make it difficult, and sometimes impossible, to synthesise results that are directly relevant to the study outcomes, interventions, and populations. In a PMA, studies are included as they are being planned or are ongoing, allowing researchers to agree on how to conduct their studies and collect common core outcomes. The PMA design enables the generation of evidence that is directly relevant to the research questions and thus increases confidence in the strength of the statements and recommendations derived from the PMA.

The ability to harmonise varies depending on the time when the PMA is first planned (fig 4). In a de novo PMA, studies are planned as part of a PMA. For PMAs of interventional studies, a de novo PMA is similar to a multicentre trial: the included trials often share a common protocol, and usually the study population, interventions, and outcome collection are fully harmonised. In contrast, some PMAs identify studies for inclusion when data collection has already finished but no analyses related to the PMA research question have been conducted (outside of data safety monitoring committees). These types of PMAs allow little to no data harmonisation and are more similar to traditional retrospective meta-analyses. Yet they still have the advantage of reducing selection bias as the studies are deemed eligible for inclusion before their PMA specific results are known.

Harmonisation of studies in a PMA can occur for different elements of the included studies: study populations and settings; interventions or exposures (that is, independent variables); and outcomes collection. For study populations, settings, and interventions/exposures, harmonisation of studies to some degree is often beneficial to enable their successful synthesis. But some variation in the individual study protocols, populations, and interventions/exposures is often desirable to improve the generalisability (that is, external validity) of the research findings beyond one study, one form of the intervention, or narrow study specific populations. The variation in populations also enables subgroup analyses, evaluating if differences in populations between and within the studies leads to

Scenario 1: PMA of concurrently conducted trials

Depending on time point of PMA formation, different levels of harmonisation are possible:

- De novo PMA is comparable to a multi-centre trial in that the combined analysis and trials are planned concurrently. Harmonisation of intervention design, study population and outcome collection possible - PMA of (mostly) completed trials is more comparable to a traditional meta-analysis. Generalisability and power are high, but there may be problems with higher heterogeneity. Yet, it is still prospective and thus risk of bias is reduced

- PMA cut-off indicates the last possible time point for a PMA to be planned to still be prospective. PMA are possible at any time point before analysis of individual studies. The earlier a PMA is planned the more harmonisation is possible

Scenario 2: PMA of trials at different stages

Depending on time point of PMA formation, different levels of harmonisation are possible:

- De novo PMA possible but may involve long waits until enough trials are completed

- PMA of a combination of planned and completed trials involves inclusion of trials at different stages. Allows harmonisation of later trials but not of trials that have already been completed

- PMA cut-off indicates the last possible time point for a PMA to be planned to still be prospective

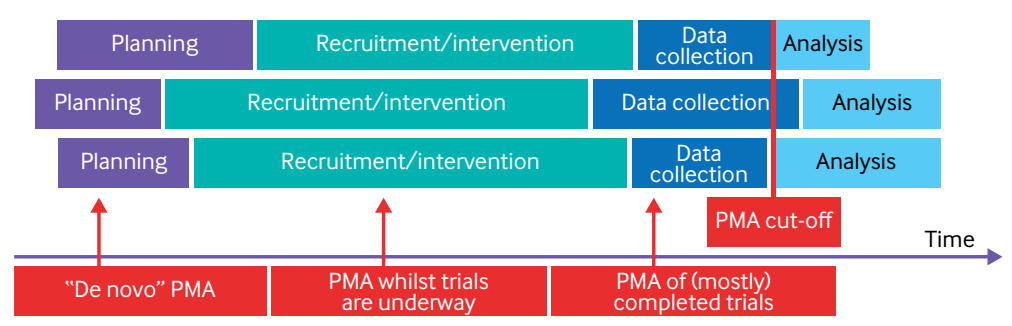

Scenario 3: PMA of observational studies at different stages The extent of possible harmonisation depends on the types of observational studies that get included: For analyses of secondary data the harmonisation of data collection is not always possible. The earlier in the study process a PMA is planned, the more harmonisation of data collection is possible:

- De novo PMA of observational studies is planned before any data are collected for any of the included studies

- PMA of on going observational studies is possible when no analysis related to the PMA research question has been performed for any of the included studies

- PMA cut-off indicates the last possible time point for a PMA to be planned to still be prospective
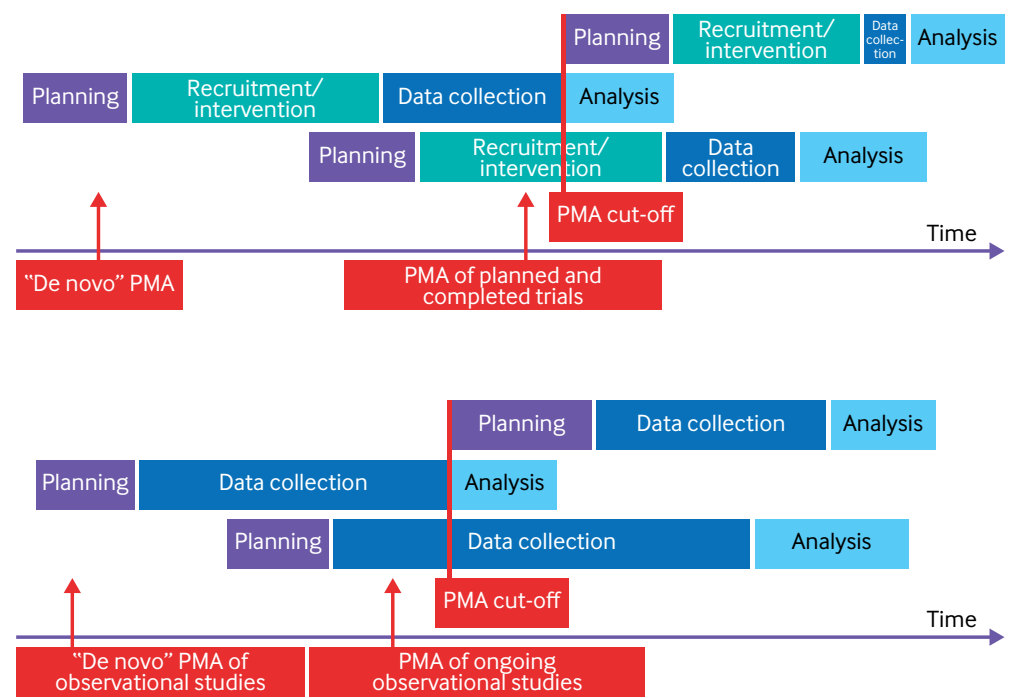

Fig 4 | Different scenarios and time points when studies can be included in a prospective meta-analysis (PMA) 
differences in treatment effects. If particular subgroups appear in more than one study, additional statistical power for subgroup analyses is also achieved.

Harmonisation of outcome collection requires careful consideration of the amount of common data needed to answer the relevant research questions. These discussions should aim to minimise unnecessary burden on participants and reduce research waste by avoiding excessive data collection, while increasing the ability to answer important research questions. Researchers can also agree to collect and analyse rare outcomes, such as severe but rare adverse events, that their individual studies would not have had the statistical power to detect. Collaborations should be specific on exactly how shared outcomes will be measured to avoid heterogeneity in outcome collection and difficulties in combining data. The COMET (core outcome measures in effectiveness trials) initiative (www.comet-initiative.org/) has introduced methods for the development of core outcome sets, as detailed in its handbook. ${ }^{24}$ These core outcome sets specify what and how outcomes should be measured by all studies of specific conditions to facilitate comparison and synthesis of the results. For health conditions with common core outcome sets, PMA collaborators should include the core outcomes, and also consider collecting other common outcomes that are particularly relevant for the specific research question posed. Not all outcomes have to be harmonised and collected by all studies: individual studies in a PMA have more autonomy than individual centres in a multicentre study and can collect study specific outcomes for their own purposes.

The improved availability of common core outcomes in a PMA has recently been shown in a PMA of childhood obesity interventions. ${ }^{25}$ Harmonisation increased from $18 \%$ of core outcomes collected by all trials before the trial investigators agreed to collaborate, to 91\% after the investigators decided to collaborate in a PMA.

\section{Case study}

Investigators of the five NeOProM trials first met in 2005 when the first trial was about to begin and the other four studies were in the early planning stages. With de novo PMA planning, all trials had the same intervention and comparator and collected similar outcome and subgroup variables. Some inconsistencies in outcome definitions and assessment methods across studies remained, however, and required substantial discussion to harmonise the final outcome collection and analyses.

\section{Step 6: synthesising the evidence and assessing} certainty of evidence

When all the individual studies have been completed, data can be synthesised in a PMA. For aggregate data PMA, results are extracted from publications or provided by the study authors. For individual participant data PMA, the line by line data from each participant in each study must be collated, harmonised, and analysed. This process is usually easier for
PMAs than for traditional, retrospective individual participant data meta-analyses because if outcome collection and coding were previously harmonised, fewer inconsistencies should arise. If possible, plans to share data should be outlined in each study's ethics application and consent form. For PMAs that are planned after the eligible studies have commenced, amendments to ethics applications may be necessary for data sharing. To assure independent data analysis, some PMAs appoint an independent data manager and statistician who have not been involved in any of the studies. The initial time intensive planning and harmonisation phase is followed by a waiting period when all the individual studies are completed before their data are made available and synthesised. During this middle period, PMAs usually demand little time and can run alongside other projects.

For studies where data safety monitoring committees are appropriate, it might be sensible for the committees to communicate and plan joint interim analyses to take account of all the available evidence when making recommendations to continue or stop a study. The PMA collaboration should consider establishing a joint data monitoring committee to synthesise data from all included studies at prespecified times. Methods for sequential meta-analysis and adaptive trial design could be considered in this context. ${ }^{26}$

When all studies have been synthesised, the methodological quality of the included studies needs to be appraised with validated tools, such as those recommended by Cochrane. ${ }^{27} 28$ The certainty of the evidence can be assessed with the grading of recommendations assessment, development and evaluation (GRADE) approach. ${ }^{29}$

\section{Case study}

The NeOProM Collaboration was established in 2005, the first trial commenced in 2005, the last trial's results were available in 2016, and the final combined analysis was published in 2018. At the request of two of the trials' data monitoring committees, an interim analysis of data from these two trials was undertaken in 2011 and both trials were stopped early. ${ }^{30}$ The five trials included in NeOProM were assessed for risk of bias with the Cochrane domains, ${ }^{31}$ and consensus was reached by discussion with the full study group. The risk of bias assessments were more accurate and complete after detailed discussion of several domains (eg, allocation concealment and blinding) between the NeOProM Collaborators than would have been possible with their publications alone. GRADE assessments were performed and published in the Cochrane version of the meta-analysis. ${ }^{32}$

\section{Step 7: interpretation and reporting of results}

Generally, the quality of the evidence derived from a PMA, and the extent to which causal inferences can be made, directly depend on the type and quality of the studies included in a PMA. The prospective nature of interventional PMAs make them similar to large multicentre trials, allowing for causal conclusions to 


\section{Table 1 | Advantages and disadvantages of a prospective meta-analysis (PMA) compared with a multicentre study and a retrospective meta-analysis Evaluation criteria PMA \\ Type of study Any that are hypothesis driven and not exploratory: interventional studies (including RCTs, non-randomised studies) and observational studies (including cohort, case-control, and cross sectional) \\ Type of data analysed Aggregate data or IPD

\begin{tabular}{ll} 
Registration & PROSPERO \\
\hline Research topics & High priority research questions, for which new studies are
\end{tabular} expected to emerge, and limited previous evidence exists; emerging, hot topics/new treatments with some preliminary evidence; areas where uncertainty remains with no agreed \\ Multicentre study \\ Any, including: interventional studies \\ (including RCTs, non-randomised studies) and observational studies (including cohort, case-control, and cross sectional) IPD \\ WHO clinical trial registry \\ Any topic with insufficient available \\ evidence to derive a reliable conclusion \\ Retrospective meta-analysis \\ Any: interventional studies (including RCTs, non-randomised studies) and observational studies (including cohort, case-control, and cross sectional) \\ Aggregate data or IPD \\ PROSPERO, Cochrane Library \\ Any topic with insufficient available evidence to derive a reliable conclusion, where studies exist that are similar enough to be meta-analysed} best practice; need power to answer important question, not able to fund one large multicentre study

\begin{tabular}{ll}
\hline Publication bias & Low risk \\
\hline $\begin{array}{l}\text { Selective reporting } \\
\text { bias }\end{array}$ & Low risk \\
\hline $\begin{array}{l}\text { Bias through } \\
\text { selection of eligibility } \\
\text { criteria and main }\end{array}$ & Low risk given no knowledge of results at study design stage \\
outcomes &
\end{tabular}

\section{Outcome}

harmonisation

Common core outcomes agreed, if studies are included in a PMA before their outcome collection. Additional rare but important outcomes can be collected that individual studies

pone would not have sufficient power to analyse Ability for individual studies to analyse additional, trial specific outcomes is maintained

\section{Population Possible for de novo PMAs; consider trade-off between}

harmonisation generalisability and harmonisation

Intervention/ Possible for de novo PMAs; consider trade-off between exposure/predictor generalisability and harmonisation

harmonisation

Statistical power

Higher power to include and analyse rare outcomes, a priori power calculations enable acquisition of optimal sample size if sufficient studies can be included in the PMA

Collaboration

High level required, particularly for IPD PMAs. If investigators refuse, aggregate data can still be included

$\begin{array}{ll}\text { Flexibility } & \text { High: decentralised, allow study specific protocol } \\ & \text { variations and outcomes that are locally relevant; new trials } \\ & \text { and intervention groups can be added throughout the study; } \\ & \text { investigators maintain autonomy }\end{array}$

Generalisability

Can be more generalisable than multicentre study because some variation is allowed in design, setting, populations, an protocols; consider trade-off between generalisability and harmonisation

Cost Low to medium: less than a multicentre study; efficient because makes best use of data but more than a retrospective meta-analysis as it demands additional planning and coordination

Duration Can be lengthy because of the waiting period while individual studies are being completed and published

Time commitment for High at outset (protocol and SAP development, establishing coordinating centre collaboration), low during middle stages (waiting period while studies are completed), high at end (analysis, publication)

Incorporation of existing evidence

Ability to update Possible, with a nested PMA design

Yes, can add studies at any time before any results are known. Can also have a living PMA where new studies are added until the research question is definitively answered

\section{Subgroup analyses Variation in populations enables subgroup analyses,} evaluating if differences in populations between and within the studies leads to differences in treatment effects. Ability to predefine important subgroups

\begin{tabular}{ll}
\hline Interpretation/ & Interventional PMA: permits causal inference. \\
reporting & $\begin{array}{l}\text { Observational PMA: associations (unless modern methods for } \\
\text { causal inference applied) }\end{array}$
\end{tabular}
plan.

Medium risk, mitigated by prospective

publication/registration of protocol

Low risk given no knowledge of results at study design stage

Prespecified in shared protocol, usually the same outcomes are collected across all sites

High risk

High risk

Moderate risk as knowledge of existing study results might influence criteria for study selection, definition of review question, treatments, and participant groups evaluated, and outcomes to be assessed

Commonly outcomes are collected, measured, or reported differently across studies, sometimes limiting ability to synthesise data. Some harmonisation possible for IPD meta-analyses, with algorithmic transformations of centrally collated data

Prespecified in shared protocol although Not possible. Population must fit predefined may be differences across sites eligibility criteria, otherwise excluded from analyses

Prespecified in shared protocol although Not possible. Intervention must fit predefined may be some variability across sites eligibility criteria, otherwise excluded from analyses

Can be limited because of inability to

A priori power calculations to obtain correct sample size possible. Sometimes power is limited in individual studies synthesise non-harmonised outcomes, or if insufficient studies are available because of funding restrictions or recruitment issues

High collaboration within study, usually Minimal

little collaboration with other related studies: research environment traditionally is competitive rather than collaborative

Medium/low: investigators must follow

Low: evidence acquisition is retrospective

single shared protocol, have less

autonomy; cannot incorporate new similar studies (unless they decide to collaborate in a PMA) other than substudies

Can have high external validity if variation Generalisability usually high but heterogeneity across sites but all follow one shared protocol as a result of uncontrollable study variation may reduce certainty of evidence

High

Low

Dependent on follow-up duration; generally quicker than a PMA

High throughout

Can be done relatively quickly

High throughout (but generally much less time needed to complete than for a PMA or multicentre study)

No, other than to inform sample size calculations or in bayesian analysis Yes

No

Yes, this can be formalised with living systematic review methodology

May have less variability in population, limiting power for subgroup analyses.

Less variability in population, limiting power for subgroup analyses. Subgroup information may not have been collected in a way that enables data synthesis

Interventional: permits causal inference. Observational study design permits

Observational: associations (unless modern associations only methods for causal inference applied) 
be drawn rather than only associations, as sometimes suggested for traditional retrospective meta-analyses. The results of observational PMAs should generally be interpreted as providing associations, not causal effects, as only the results of observational studies are included. But with modern methods for causal inference from observational studies, justification for supporting conclusions about causality can sometimes be found. ${ }^{33}$

Currently no PMA specific reporting standards exist, but where applicable, PMA authors should follow the PRISMA-IPD (PRISMA of individual participant data) statement ${ }^{34}$ if they are reporting an individual participant data PMA, or the PRISMA statement ${ }^{35}$ if they are reporting an aggregate data PMA. As well as the PRISMA items, authors of PMAs need to report on identification of planned and ongoing studies, the PMA timeline, collaboration policies, and outcome harmonisation processes.

Discussions about methodology and interpretation of the results among all collaborators can sometimes be difficult to navigate, particularly if the results from the combination of the studies contradict the results of some of the individual studies. Although these discussions can be demanding and time consuming, robust discussion among experts can lead to well considered and high quality publications that can directly inform policy and practice.

For the successful management of a PMA collaboration, an explicit authorship policy should be in place. One model is to offer authorship to each member of the secretariat, and one investigator from each included study, for the main PMA publication, assuming they fulfil the authorship criteria of the International Committee of Medical Journal Editors (ICMJE). This model incentivises ongoing involvement and allows for multiple viewpoints to be integrated in the final publication. The collaborators usually agree that the final PMA results cannot be published until the results of each study are accepted for publication, but this is not essential.

\section{Case study}

At least one investigator from each of the participating trials was a co-author on the final publication for NeOProM. ${ }^{13}$ Collaborators met regularly, face to face and by phone, to resolve opposing views and achieve consensus on the interpretation of the PMA findings. Face to face meetings were crucial in resolving major disagreements within the NeOProM Collaboration. The collaborators used the PRISMA-IPD checklist for reporting of the PMA.

\section{Discussion}

PMAs have many advantages: they help reduce research waste and bias, while greatly improving use of data, and they are adaptive, efficient, and collaborative. PMAs increase the statistical power to detect effects of treatment and enable harmonised collection of core outcomes, while allowing enough variation to obtain greater generalisability of findings.
Compared with a multicentre study, PMAs are more decentralised and allow greater flexibility in terms of funding and timelines. Compared with a retrospective meta-analysis, PMAs enable more data harmonisation and control. Planning a PMA can help a group of researchers prioritise a research question they can address collaboratively and determine the optimal sample size a priori. Disadvantages of PMAs include difficulties in searching for planned and ongoing studies, often long waiting periods for studies to be completed, and difficulties in reaching consensus on the interpretation of the results. Table 1 shows a detailed comparison of the features and advantages and disadvantages of PMAs, multicentre studies, and retrospective meta-analyses.

\section{Integration of PMAs with other next generation systematic review methodologies}

PMAs can be combined with other new systematic review methodologies. Living systematic reviews begin with a traditional systematic review but have continual updates with a predetermined frequency. Living systematic reviews address similar research questions as PMAs (high priority questions with inconclusive evidence in an active research field). ${ }^{36}$ In some instances it might be beneficial to combine these two methodologies. If authors are considering a PMA in a discipline where evidence is expected to become available gradually, a living PMA is an option. In living PMAs, new studies are included as they are being planned (but importantly before any of the results related to the PMA research questions are known), until a definitive effect has been found or the maximum required statistical information has been reached to conclude that no clinically important effect has been found. ${ }^{37}$ Appropriate statistical methods for multiple testing should be strongly considered in living PMAs, such as sequential meta-analysis methodology which controls for type 1 and type 2 errors and takes into account heterogeneity. ${ }^{26}$ PMA methodology can also be combined with other methods, such as network meta-analysis or meta-analysis of prognostic models.

\section{Future for PMAs}

With the advancement of machine learning, artificial intelligence, and big data, new horizons are seen for PMAs. Several steps need to be taken to improve the feasibility and quality of PMAs. Firstly, the ability to identify planned and ongoing studies needs to be improved by introducing further mechanisms to promote and enforce study registration and providing guidance on the best search strategies. The ICMJE requirement for prospective registration of clinical trials, together with several other ethical and regulatory initiatives, has improved registration rates of clinical trials but more improvement is needed. ${ }^{38}$

22 Possible solutions include the integration of data submitted to ethics committees, funding bodies, and clinical trial registries. ${ }^{21}$ The Cochrane PMA Methods Group, in collaboration with several trial registries, is working on improving methods for identifying 
planned and ongoing studies. Future technologies might automate the searching and screening process for planned and ongoing studies and automatically connect researchers who are planning similar relevant studies. Furthermore, the reporting and quality of PMAs needs to be improved. The reporting of PMAs would be greatly helped by the development of a standardised set of reporting guidelines to which PMA authors can adhere. Such guidelines are currently under development. Also, the development of PMA specific evidence rating tools (such as an extension to the GRADE approach) would be highly desirable. The Cochrane PMA Methods Group will publicise any new developments in this area on their website (https:// methods.cochrane.org/pma/).

\section{Conclusion}

PMAs have many advantages, and mandating trial registration, development of core outcome sets, and improved data sharing abilities have increased opportunities for conducting PMAs. We hope this step by step guidance on PMAs will improve the understanding of PMAs in the research community and enable more researchers to conduct successful PMAs. The Cochrane PMA Methods Group can offer advice for researchers planning to undertake PMAs.

Contributors: ALS conceived the idea and facilitated the workshop and discussions. LA, DG, KEH, and ALS participated in the workshop, and JAB and SC contributed to further discussions after the workshop. ALS, SC, and KEH performed the searches for a scoping review that was conducted in preparation for this article, reviewing all prospective meta-analyses and methods papers on prospective meta-analyses in health research to date. LA was the coordinator of the NeOProM Collaboration and KEH was a member. ALS wrote the first draft of the manuscript. All authors contributed to and revised the manuscript. ALS is the guarantor.

Competing interests: We have read and understood the BMJ Group policy on declaration of interests and declare the following: all authors are convenors or members of the Cochrane PMA Methods Group and have been involved in numerous prospective meta-analyses. LA $D G$, and JAB have published several methods articles on prospective meta-analyses and are authors of the prospective meta-analysis chapter in the Cochrane Handbook for Systematic Reviews of Interventions. LA manages the Australian New Zealand Clinical Trials Registry (ANZCTR). ALS and KEH work for the ANZCTR. JAB is a full time employee of Johnson \& Johnson.

Provenance and peer review: Not commissioned; externally peer reviewed.

This is an Open Access article distributed in accordance with the Creative Commons Attribution Non Commercial (CC BY-NC 4.0) license, which permits others to distribute, remix, adapt, build upon this work non-commercially, and license their derivative works on different terms, provided the original work is properly cited and the use is noncommercial. See: http://creativecommons.org/licenses/by-nc/4.0/.

1 National Health and Medical Research Council. NHMRC additional levels of evidence and grades for recommendations for developers of guidelines. National Health and Medical Research Council, 2009.

2 loannidis JP. The mass production of redundant, misleading, and conflicted systematic reviews and meta-analyses. Milbank Q 2016:94:485-514. doi:10.1111/1468-0009.12210

3 Chan AW, Krleza-Jerić K, Schmid I, Altman DG. Outcome reporting bias in randomized trials funded by the Canadian Institutes of Health Research. CMAl 2004;171:735-40. doi:10.1503/cmaj.1041086

4 Simes RJ. Publication bias: the case for an international registry of clinical trials. J Clin Oncol 1986;4:1529-41. doi:10.1200/ JCO.1986.4.10.1529

5 Berlin JA, Ghersi D. Preventing Publication Bias: Registries and Prospective Meta-Analysis. Publication Bias in Meta-Analysis. John Wiley \& Sons, 2006:35-48.
6 Askie L, Offringa M. Systematic reviews and meta-analysis. Semin Fetal Neonatal Med 2015;20:403-9. doi:10.1016/j. siny.2015.10.002

7 loannidis J. Next-generation systematic reviews: prospective metaanalysis, individual-level data, networks and umbrella reviews. $\mathrm{Br}$ Sports Med 2017:51:1456-8. doi:10.1136/bjsports-2017-097621

8 loannidis JP. Meta-research: The art of getting it wrong. Res Synth Methods 2010;1:169-84. doi:10.1002/jrsm.19

9 Halpern SD, Karlawish JHT, Berlin JA. The continuing unethical conduct of underpowered clinical trials. JAMA 2002;288:358-62. doi:10.1001/jama.288.3.358

10 Ghersi D, Berlin J, Askie L. Prospective metaDanalysis. In: Higgins JPT, Green S (eds), Cochrane Handbook for Systematic Reviews of Interventions Version 5.1.0 (updated March 2011). The Cochrane Collaboration, 2011:559-70.

11 Margitić SE, Morgan TM, Sager MA, Furberg CD. Lessons learned from a prospective meta-analysis. J Am Geriatr Soc 1995;43:435-9. doi:10.1111/j.1532-5415.1995.tb05820.x

12 Probstfield I, Applegate WB. Prospective meta-analysis: ahoy! A clinical trial?] Am Geriatr Soc 1995;43:452-3. doi:10.1111/j.1532-5415.1995.tb05823.x

13 Askie LM, Darlow BA, Finer N, et al, Neonatal Oxygenation Prospective Meta-analysis (NeOProM) Collaboration. Association between oxygen saturation targeting and death or disability in extremely preterm infants in the neonatal oxygenation prospective meta-analysis collaboration. JAMA 2018;319:2190-201. doi:10.1001/jama.2018.5725

14 Cole CH, Wright KW, Tarnow-Mordi W, Phelps DL, Pulse Oximetry Saturation Trial for Prevention of Retinopathy of Prematurity Planning Study Group. Resolving our uncertainty about oxygen therapy. Pediatrics 2003;112:1415-9. doi:10.1542/peds.112.6.1415

15 Green S, Higgins JPT (eds). Preparing a Cochrane review. In: Cochrane Handbook for Systematic Reviews of Interventions Version 5.1.0 (updated March 2011). The Cochrane Collaboration, 2011.

16 Moher D, Shamseer L, Clarke M, et al, PRISMA-P Group. Preferred reporting items for systematic review and meta-analysis protocols (PRISMA-P) 2015 statement. Syst Rev 2015;4:1. doi:10.1186/20464053-4-1

17 Booth A, Clarke M, Dooley G, et al. The nuts and bolts of PROSPERO an international prospective register of systematic reviews. Syst Rev 2012;1:2. doi:10.1186/2046-4053-1-2

18 Askie LM, Brocklehurst P, Darlow BA, Finer N, Schmidt B, TarnowMordi W, NeOProM Collaborative Group. NeOProM: Neonatal Oxygenation Prospective Meta-analysis Collaboration study protocol. BMC Pediatr 2011;11:6. doi:10.1186/1471-2431-11-6

19 World Health Organization (WHO). WHO International Clinical Trials Registry Platform (ICTRP) Search Portal: http://apps.who.int/ trialsearch/ [accessed 6 November 2018].

20 Isojarvi J, Wood H, Lefebvre C, Glanville J. Challenges of identifying unpublished data from clinical trials: Getting the best out of clinical trials registers and other novel sources. Res Synth Methods 2018;9:561-78.

21 Hunter KE, Seidler AL, Askie LM. Prospective registration trends, reasons for retrospective registration and mechanisms to increase prospective registration compliance: descriptive analysis and survey. BMJ Open 2018;8:e019983. doi:10.1136/ bmjopen-2017-019983

22 Dal-Ré R, Ross JS, Marušić A. Compliance with prospective trial registration guidance remained low in high-impact journals and has implications for primary end point reporting / Clin Epidemiol 2016;75:100-7. doi:10.1016/j.jclinepi.2016.01.017

23 Harriman SL, Patel J. When are clinical trials registered? An analysis of prospective versus retrospective registration. Trials 2016;17:187. doi:10.1186/s13063-016-1310-8

24 Williamson PR, Altman DG, Bagley H, et al. The COMET Handbook: version 1.0. Trials 2017;18(Suppl 3):280. doi:10.1186/s13063 017-1978-4

25 Seidler A, Hunter K, Mihrshahi S, et al. Lessons learned from a prospective meta-analysis (PMA) of childhood obesity prevention quantifying the advantages of a PMA and future recommendations. In: Abstracts of the 25th Cochrane Colloquium, Edinburgh, UK. Cochrane Database Syst Rev 2018;9(Suppl 1):CD201801. https:// abstracts.cochrane.org/2018-edinburgh/lessons-learnedprospective-meta-analysis-pma-childhood-obesity-prevention$\%$ E2\%80\%93 [accessed 11 September 2019]

26 Simmonds M, Salanti G, McKenzie J, Elliott J, Living Systematic Review Network. Living systematic reviews: 3. Statistical methods for updating meta-analyses. J Clin Epidemiol 2017;91:38-46. doi:10.1016/j.jclinepi.2017.08.008

27 Higgins JPT, Sterne JAC, Savović J, et al. A revised tool for assessing risk of bias in randomized trials. Cochrane Database Syst Rev 2016;10(Suppl 1):29-31.

28 Sterne JA, Hernán MA, Reeves BC, et al. ROBINS-I: a tool for assessing risk of bias in non-randomised studies of interventions. BM/ 2016;355:14919. doi:10.1136/bmj.i4919 
29 Guyatt G, Oxman AD, Akl EA, et al. GRADE guidelines: 1. IntroductionGRADE evidence profiles and summary of findings tables. I Clin Epidemiol 2011;64:383-94. doi:10.1016/j.jclinepi.2010.04.026

30 Stenson B, Brocklehurst P, Tarnow-Mordi WU.K. BOOST II trial, Australian BOOST II trial, New Zealand BOOST II trial. Increased 36-week survival with high oxygen saturation target in extremely preterm infants. N Engl / Med 2011;364:1680-2. doi:10.1056/ NEJMc1101319

31 Higgins J. Altman D. Sterne J. Assessing risk of bias in included studies. In: Higgins J, Green S, eds. Cochrane Handbook for Systematic Reviews of Interventions Version 5.1.0. [updated March 2011] The Cochrane Collaboration, 2011.

32 Askie LM, Darlow BA, Davis PG, et al. Effects of targeting lower versus higher arterial oxygen saturations on death or disability in preterm infants. Cochrane Database Syst Rev 2017;4:CD011190. doi:10.1002/14651858.CD011190.pub2

33 Pearl J. Causality. Cambridge University Press, 2009. doi:10.1017/ CB09780511803161

34 Stewart LA, Clarke M, Rovers M, et al, PRISMA-IPD Development Group. Preferred reporting items for a systematic review and meta- analysis of individual participant data: the PRISMA-IPD statement. JAMA 2015;313:1657-65. doi:10.1001/jama.2015.3656

35 Moher D, Liberati A, Tetzlaff J, Altman DG, PRISMA Group. Preferred reporting items for systematic reviews and meta-analyses: the PRISMA statement. PLoS Med 2009;6:e1000097. doi:10.1371/ journal.pmed.1000097

36 Elliott JH, Synnot A, Turner T, et al, Living Systematic Review Network. Living systematic review: 1. Introduction-the why, what, when, and how. J Clin Epidemiol 2017;91:23-30. doi:10.1016/j. jclinepi.2017.08.010

37 Guyatt GH, Oxman AD, Kunz R, et al. GRADE guidelines 6. Rating the quality of evidence--imprecision. J Clin Epidemiol 2011;64:1283-93. doi:10.1016/j.jclinepi.2011.01.012

38 Tan AC, Jiang I, Askie L, Hunter K, Simes RJ, Seidler AL. Prevalence of trial registration varies by study characteristics and risk of bias. J Clin Epidemiol 2019;113:64-74. doi:10.1016/j.jclinepi.2019.05.009

Web appendix 1: Prospective meta-analysis (PMA) scoping review methods 